\title{
A Review on Usage Based Insurance System Using RFID Cards
}

\author{
Arun G.* , Anush M., Amruth M. Rai and Geetha M.N. \\ Department of Electronics and Communication Engineering, Vidyavardhaka College of Engineering, \\ Mysuru 570002,Karnataka, India; arunruna082@gmail.com, anushmurali.06@gmail.com, \\ amruthmrai007@gmail.com, geetha.mn@vvce.ac.in
}

\begin{abstract}
Objectives: This study reviews the usage based insurance system for automobiles using RFID cards. Method analysis: Usage based insurance is a system where the premium is estimated based on the usage behavior of the customer. Findings/ application: This method is mainly developed to improve the method of automobile insurance in accordance with the driving behavior of the driver and after considering the main parameters required to drive a vehicle safely. This method is mainly based on usage based insurance (UBI) system which is also popularly known as pay as you drive (PAYD) or pay how you drive (PHYD). People many times feel burdened on the amount of premium one has to pay.
\end{abstract}

Keywords: Usage Based Insurance (UBI), Pay As YOU Drive (PAYD), Pay-How-You-Drive (PHYD), RFID Cards

\section{Introduction}

Vehicle insurance is an important document one must have to use your vehicles on public roads. Most governments have rules which make it mandatory that the vehicle plying on the public roads have insurance. It is also beneficial for the riders as in the case of any untoward incidents one can rest assured that any damages can be recovered without a sudden shock to your income. At the same time, this poses a serious challenge to the insurance providers to calculate the most accurate premium for any customer. Most insurance providers will just consider the demographic parameters like age, sex, location etc. and decide on the premium. The driving behavior of the customer is not considered in calculating the premium. Taking the driving behavior into account may lead to better driving practices being adopted by the drivers to reduce the premium on their vehicles. The driving practices need to be monitored on a regular basis to understand how the driver is driving the vehicle. This can be done by collecting data on the various driving parameters by placing appropriate sensors in the vehicle and analyzing the data obtained from the sensors.

Here, in this method, the driver of the vehicle is provided with an RFID card which is required to start the vehicle. A vehicle analyzing all the parameters required such as the acceleration of the vehicle, inbuilt GPS tracker system to know where and how fast the vehicle is moving and at what time, it also checks whether the driver has worn his helmet or not when the driver exceeds his speed limit considering all the parameter the insurance premium he has to pay is calculated. This is mainly developed to improve the method of automobile insurance in accordance with the driving behavior of the driver and after considering the main parameters required to drive a vehicle safely. At the time of his insurance's expiry, the driver gets the notification of his vehicle's insurance and the amount that has to be paid. The driver can get all the information about his vehicle through his card.

UBI - Usage based insurance.

GPS - Global positioning system.

RFID - Radio frequency identification

*Author for correspondence 


\section{Literature Survey}

RFID tags are used for tracking of the vehicles 1,2 in traffic discuss in their paper the proper tracking systems for vehicles using RFID tags on all vehicles and using this tracking log to analyze and reduce the traffic congestion on the various routes of cities. The traffic signal timings at the junctions can be controlled based on the traffic density on the respective roads. Data obtained from vehicle tracking can also be used to divert the traffic from heavily congested routes to low congested routes.

In, $\underline{\underline{3}}$ a machine controlled traffic to direct a smart system to prevent trafficking of vehicles, detection of a stolen vehicle and ambulance headway is discussed. In this system, every vehicle is tagged with a unique RFID card. An RFID reader is placed on the road, it will count the number of vehicles tagged with the RFID card when the vehicle crosses the reader at a given time. The count of vehicles is detected by the IR sensor and location is tracked by using the GSM module. When the traffic density crosses a certain pre-defined threshold value the green light is turned on and the vehicles are allowed to pass. This will assist in the reduction of traffic congestion. If the RFID reader comes across a stolen vehicle it will immediately alert the nearby police station. This system also can detect emergency service vehicles like ambulances, and provide them clear passage.

The theft of vehicles is a major problem and the value of the stolen vehicle is usually very large. The insurance premium to be paid by each of the customers will increase over time if the incidence of vehicle theft keeps on increasing. Thus it would be beneficial to incorporate systems that will prevent theft of vehicles and in the instance of theft quick and efficient tracking, detection and recovery of the stolen vehicle. RFID based vehicle immobilizer systems have been studied by various researchers, $\underline{\underline{4}-\underline{6}}$ presents the RFID used as a vehicle immobilizer system. It features a low hacking probability to preserve the safety of the passenger of the hijacked vehicles. The concept used is a tag with a significantly large character set. The receiver unit is smartly integrated to bring the vehicle speed down to zero and immobilize it. It provides a safe and valid solution for the ever-increasing threat in the vehicle hijacking.

Vehicle monitoring and tracking is an essential part of any usage based insurance system. Vehicle monitoring systems are required to monitor the location, and other parameters like speed, fuel consumption, temperature, engine health, braking systems, tire pressure etc. of the vehicle. Various vehicle monitoring systems have been studied earlier, $-\underline{\underline{7}-\underline{9}}$ the basic objective is the extension of a vehicle monitoring system for all the registered vehicles using RFID cards. It uses a 13.56 Mhz RFID module and sends SMS warning by using a GSM module, and an integrated ultrasonic sensor to identify and detect the systems (vehicles) entering the zone and an integrated Arduino camera to capture the images.

In,,$\underline{10}$ the main objective is to aim at lane-level arranging on the road even in different circumstances. RFID tags will be attached to the entrances of the lanes to manifest its lane number. When travelling onto a lane the vehicle can obtain its initial lane number using the RFID tag reader. Using the lane refereeing sub-system, the horizontal placement error of GPS can be reviewed. Hence the lanelevel position identification with longitudinal accuracy of several meters can be attained and used for real-time safety and self-driving on open roads such as highways can be done.

The GPS signals are very sensitive to ground and interference, they may become inaccessible in certain areas. It becomes very difficult to get an accurate location of vehicles in those areas. An error cognitive positioning system using RFID tags is implemented. $\underline{11}$ Passive RFID tags are placed on the roads at specific locations and the corresponding tag readers are placed on the vehicles. The accurate position of the vehicle is obtained by the errorcognitive positioning system. This method gives a costeffective way to pinpoint a vehicle with sub-meter error on average with lightly installed RFID tags.

Identification of vehicles in a hit and run case and collision is sometimes very difficult. RFID based vehicle identification system using collision sensors to detect a collision between vehicles have been reported. In $\underline{12}, \underline{13}$ a hit and run situation the collision sensors detect the collision, the time and location of the collision and using the RFID tag the vehicles involved can be identified. This will help the insurance companies to identify the vehicle owners and the rash drivers involved in the hit and run case. Which eventually will be useful evidence to prove the case in court or to obtain the appropriate compensation from the owner who is at fault.

Speed breakers are used to control the speed of vehicles on the road. Construction of speed breakers on the roads is a costly process. An RFID based virtual speed breakers have been proposed. $\underline{14}$ This will reduce the structure cost as well as repair cost of the vehicle. In this system, an 
RFID tag is placed in locations where the speed breakers are necessary. When the vehicle reaches the location the RFID tag transmits the information of the speed breaker to the vehicle and RFID sensor in the vehicle receives the signal and relays the information to the control unit in the vehicle, which in turn control speed and apply brakes to bring the speed of the vehicle to the desired level. It is a clever system that will protect the passengers whether the driver in the vehicle is conscious or not. This maintains maximum safety to passengers and to the public.

In, $\underline{15}$ vehicle's all risk factors like driving style, risk modes are evaluated using an on-board diagnosis device that is placed on each vehicle. Here 4 factors are included occurring, criticalness, verification and preventing, and by categorizing all these results of risk factor will be updated and given to insurance company using triangular numbers. The driver's approach of riding is evaluated from raw data and the fuzzy logic algorithm is used to get an equation and tabulated the obtained risk factors. This method is used at the beginning of usage based insurance system.

Almost every individual is using a smartphone nowadays. The smartphone is quite useful as it is embedded with many sensors and measurement systems, which can record data of various physical parameters. This data can be used to enhance or improve the livelihood of people and society. A smartphone-based measurement system and framework have been proposed which can be used for vehicle traffic monitoring and usage based insurance. $\frac{16}{16}$ Here the various parameters related to traffic and the vehicle are collected and stored in the smartphone. This data is relayed in real-time to the cloud. This data is useful to detect the traffic condition and if the traffic density is increasing then suggesting different routes to decongest the routes. The obtained data is also used to determine the driving behavior of the driver and which in turn will help in determining their insurance premium.

\section{Discussion}

Here passive RFID cards are used on roads and readers in the vehicle for tracking the device in inaccessible places. Here $\underline{13}$ when the collision happens between vehicles and is sensed, the RFID tag readers on the vehicles are triggered and they obtain the vehicle details from the respective RFID tags on the vehicle. This structure makes it easier for vehicle owners to reduce the rash drivers and thus enhance road safety. RFID based virtual speed breaker instead of the traditional speed breaker will reduce the infrastructure cost as well as vehicle repairs cost. It is a smart vehicle monitoring system that will ensure the safety of the passengers whether the driver is conscious or not. In $\frac{15}{}$ each vehicle's OBD devices will be installed, using this module the risk mode of each driver will calculate and analyzed and the data will be sent to RFID. The data stream runs the business model and part of the revenue streams, ensuring the funding of the total measurement system and creates value for the end-user and also the insurance company.

\section{Conclusion}

RFID based insurance management system would be a great addition to the insurance system at the current economic condition. The majority of the people are found to be from the middle class or from below poverty line economic background, vehicle owners from such backgrounds find it hard to pay the insurance premium, making them bailout of the insurance payment procedure. Bringing up the above management system would greatly encourage paying for insurance. In the current system, cautious and irresponsible drivers are to pay the same insurance premium as a responsible driver, which seems unjust. Since our system monitors the driving condition of the driver, the one who shows cautious driving has to pay a lesser premium, whereas reckless drivers have to pay a larger insurance premium. This thereby will increase good driving habits to be observed in drivers, reducing accidents and fatalities. Since the system monitors all the aspects of driver/ rider, from the initial step of wearing the helmet, him driving until he stops at the destination, will be greatly increasing the safety of the driver and the pedestrians also.

\section{Acknowledgement}

The authors express gratitude to Accendere Knowledge Management Services Pvt Ltd for the assistance provided in preparing the manuscript.

\section{References}

1. Pandit AA, Mundra AK, Talreja J. RFID tracking system for vehicles (RTSV). In: 2009 first international conference on computational intelligence, communication systems and networks; 2009. P. 160-65. 
2. Bajaj D, Gupta N. GPS Based automatic vehicle tracking using RFID. Int J Eng Innov Technol. 2012;1(1):31-35.

3. Sivakumar R, Vignesh G, Narayanan V, Prakash S, Sivakumar V. Automated traffic light control system and stolen vehicle detection. In: 2016 IEEE international conference on recent trends in electronics, information \& communication technology; 2016. P. 1594-7.

4. Lemke K, Sadeghi AR, Stüble C. Anti-theft protection: electronic immobilizers. In: Lemke K, Paar C, Wolf M, editors. Embedded security in cars. Berlin, Heidelberg: Springer; 2006. P. 51-67.

5. Kaiser U. Theft protection by means of embedded encryption in RFID transponders (immobilizer). In: ESCAR conference, Cologne, Germany; 2003.

6. Jayendra G, Kumarawadu S, Meegahapola L. RFID-based anti-theft auto security system with an immobilizer. In: 2007 International conference on industrial and information systems; 2007. P. 441-6.

7. Mandal K, Sen A, Chakraborty A, Roy S, Batabyal S, Bandyopadhyay S. Road traffic congestion monitoring and measurement using active RFID and GSM technology. In: 14th international IEEE conference on intelligent transportation systems; 2011.

8. Yu M, Zhang D, Cheng Y, Wang M. An RFID electronic tag based automatic vehicle identification system for traffic IoT applications. In: Chinese control and decision conference; 2011.

9. Panganiban EB, Dela Cruz JC. RFID-based vehicle monitoring system. In: 2017 IEEE 9th international conference on humanoid, nanotechnology, information technology, communication and control, environment and management; 2017. P. 1-6.

10. Bhavke A, Pai S. Smart weight based toll collection \&amp; vehicle detection during collision using RFID. In: 2017 international conference on microelectronic devices, circuits and systems; 2017. P. 1-6.

11. Qin H, Peng Y, Zhang W. Vehicles on RFID: error-cognitive vehicle localization in GPS-less environments. IEEE Trans Veh Technol. 2017;66(11):9943-57.

12. Bhavke A, Pai S. Advance automatic toll collection \& vehicle detection during collision using RFID. In: International conference on nascent technologies in engineering; 2017.

13. Jeevagan N, Santosh P, Berlia R, Kandoi S. RFID based vehicle identification during collisions. In: IEEE global humanitarian technology conference; 2014. P. 716-20.

14. Hossain MN, Hoque KA, Ullah SMR, Islam MM. RFID based virtual speed breakers: perspective Bangladesh. In: 2014 international conference on electrical engineering and information \& communication technology; 2014. P. $1-5$.

15. Nai W, Chen Y, Yi Yu, Zhang F, Dong D, Zheng W. Fuzzy risk mode and effect analysis based on raw driving data for payhow-you-drive vehicle insurance. In: IEEE international conference on big data analysis; 2016.

16. Handel P, Ohlsson J, Ohlsson M, Skog I, Nygren E. Smartphone-based measurement systems for road vehicle traffic monitoring and usage-based insurance. IEEE Syst J. 2014;8(4):1238-48. 